\title{
Performance Comparison of CDR-MAC at Linear and Random Topology for Wireless Ad Hoc Network
}

\author{
Md. Jahangeer Alam \\ Department of Computer and \\ Information Science \\ Jazan Community College \\ Jazan University, Jazan
}

\author{
Asrar Ahmad Ansari \\ College of e-Learning \& \\ Distance Learning Deanship \\ King Saud University, Riyadh \\ Saudi Arabia- 11461 \\ Zafrul Hasan \\ E- Learning and IT Unit \\ College of Nursing \\ King Saud University, Riyadh \\ Saudi Arabia- 11485
}

\author{
Khwaja Mohammad Athar \\ Siddique \\ College of Computer Science \\ Jazan University \\ Jazan, Saudi Arabia
}

\begin{abstract}
In this paper we compare the performance of Circular Directional RTS MAC (CDR-MAC) protocol at linear and random topology for wireless ad hoc network. We know that directional antenna gives higher transmission range than that of omni-directional antenna due to its higher gain. Most of the existing research on Directional MAC the nodes of the network distributed in random manner. Although with directional antenna we get better spatial reuse than that of omni-directional antenna in the network as well as higher transmission range. However, in linear topology, the network performance is degrades because large area blocks due to higher transmission range. We implement the Circular Directional RTS MAC (CDR MAC) protocol in OPNET 14.5 simulation tool and design two scenario for linear and random topology.
\end{abstract}

\section{Keywords}

Directional antenna, Circular RTS, WLAN, Ad-hoc network, Linear Topology, Random Topology..

\section{INTRODUCTION}

Wireless Ad-hoc networks have fascinate a emergent attention in many application domains, which include military applications, commercial applications, and applications in environments where the presence of an infrastructure network is difficult or not reasonable. Presently, the technology of sending data over the air is based on omni-directional antenna; therefore, most of the research in designing Medium Access Control (MAC) protocols has supposed their deployment. Thus the number of protocols, including the industry standard IEEE 802.11 [1], [2] which appears to effectively solve the challenges projected in this environment.

Directional antennas give many benefits in ad hoc network. The targeted nature of the sending data, results in spatial reuse, as there can be multiple transmissions in the same neighbourhood without the destruction of the transmitted packets. Additionally, the directional transmission increases the signal energy toward the direction of the receiver, resulting in the increase of the coverage area. These two benefits widely lead in the increase of the channel capacity [3].

Unluckily, in an ad hoc environment, directional transmissions cause some serious problems, like hidden and deafness of nodes [4], and the determination of neighbors' locations. The determination of neighbors' locations is a natural problem that stands for the transmission of a packet. The transmitter has to know the location of the receiver to points its beam in that direction.

Although, directional antenna gives better performance than that of omni-directional antenna, but in linear topology of the network, it degrades their performance due to their higher transmission range blocks large area of the network. Therefore, the network does not get more benefits of spatial reuse. In this paper we implement the CDR MAC protocol [3] in OPNET 14.5 to compare the performance results of the linear and random topology.

The rest of this paper is organized as follows: In the next section, we discuss previous related work. In Section B, we give a summary CDR MAC protocol. Section C contains the simulation details. In Section D, we compare the performance of results of the linear and random topology and finally, in Section $\mathrm{E}$, we give the conclusions.

\section{RELATED WORK}

Nasipuri et al. [5] proposed MAC implemented multiple noncircular directional antenna. In their method the RTS and CTS is transmitted omnidirectional, whereas the DATA and ACK transmission is directional. Omnidirectional RTS/CTS badly affect the spatial reuse. It causes all the over-hearer's to stop their transmissions.

In Ko et al. [6] proposed MAC, the RTS, DATA and ACK is transmitted in directional mode, while the CTS transmission is omnidirectional. Due to directional RTS there is slight improvement in spatial reuse. Both protocols suffer with deaf and hidden node problems.

Takata et al. [7] proposed a MAC for deafness avoidance in the ad hoc networks. They introduce a new term wait to send (WTS), which is transmitted after DRTS/DCTS in circular mode, whereas the DATA and ACK is transmitted directionally. In this protocol overhead is increased as a tradeoff to throughput but spatial reuse is increased as compared to above protocols. Korakis et al. [8] proposed a MAC to fully exploit the directional antennas in ad hoc networks. They use CRTS to inform entire neighborhood about future transmission. CTS, DATA and ACK are sent directionally towards RTS originator. Due to CRTS, the deafness and hidden node problems decrease but DCTS still causes some deafness and hidden node troubles.

In Jakllari et al. [9] scheme, the communicating nodes transmits circular RTS and CTS before directional DATA transmission. CRTS and CCTS reduce deafness and hidden node problems and 
enhance the reusability of spectrum. But due to circular RTS/CTS delay is increased.

Gossain et al. [10] proposed scheme decreases the shortcoming of previous MAC by sending the optimized circular RTS/CTS after the successful RTS/CTS handshake. But due to omnidirectional reception through idle nodes, the hidden node problem due to asymmetry in gain still remains. Further developed work can be found in Bazan et al. [11], where they have surveyed most of the prominent directional MAC protocols.

\section{CDR MAC PROTOCOL}

Jallari et al [12] proposes a MAC protocol that incorporates circular RTS and CTS transmissions. They shows that the circular transmission of the control messages helps avoid collisions of both DATA and ACK packets from hidden terminals. In their scheme the all transmission is directional. If a node has packet it transmits RTS towards the destined node. After transmitting the RTS, the node shifts the beam on the right and sends the same RTS message with other beam and so on. Finally, the sequential transmissions circularly cover the entire area around the transmitters. Then the sender node waits for CTS in omni directional mode. The receiver node replies with CTS towards sender node. After sending the CTS to sender, the receiver node also sends circular CTS to its vicinity similar to sender node by shifting its beam.

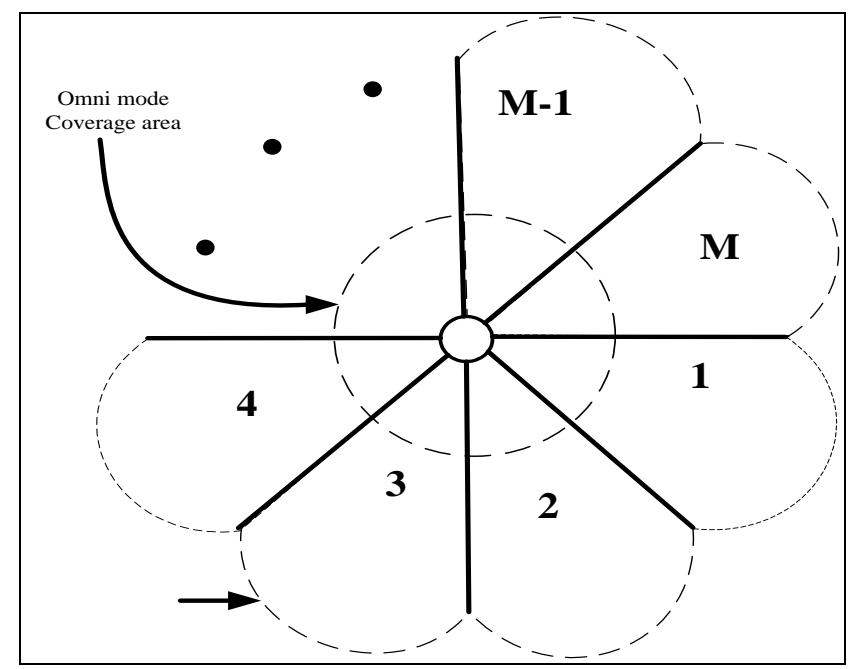

Figure. 1. Circular transmission in CDR MAC

Figure. 1 shows the working procedure of CDR MAC protocol. In the figure, a node transmits RTS to its destined node thorough beam number 3 , and then it shifts the beam to sector 2 and sends RTS again then sector 1 and so on. Finally, the nodes transmit RTS circularly around the node. The receiver node receives the RTS, because it is in omni mode, transmits CTS towards the sender, then similar to sender node its transmit CTS circularly to its vicinity, then the starts DATA transmission.

\section{SIMULATION DETAIL}

In this section we discuss about our simulation setup. For simulation we use OPNET 14.5 [13]. OPNET provides virtual environment of network scenario. It provides several editors such as antenna pattern editor, node editor, process editor and packet format editor etc. to give real scenario of the network. For our simulation, we design antenna in antenna pattern editor, node model in node editor. We implement CDR MAC protocol in OPNET 14.5 simulation tool. For simulation we attached an antenna with default node model provided by the simulation tool. The structure of node model for our simulation depicted in
Figure. 2

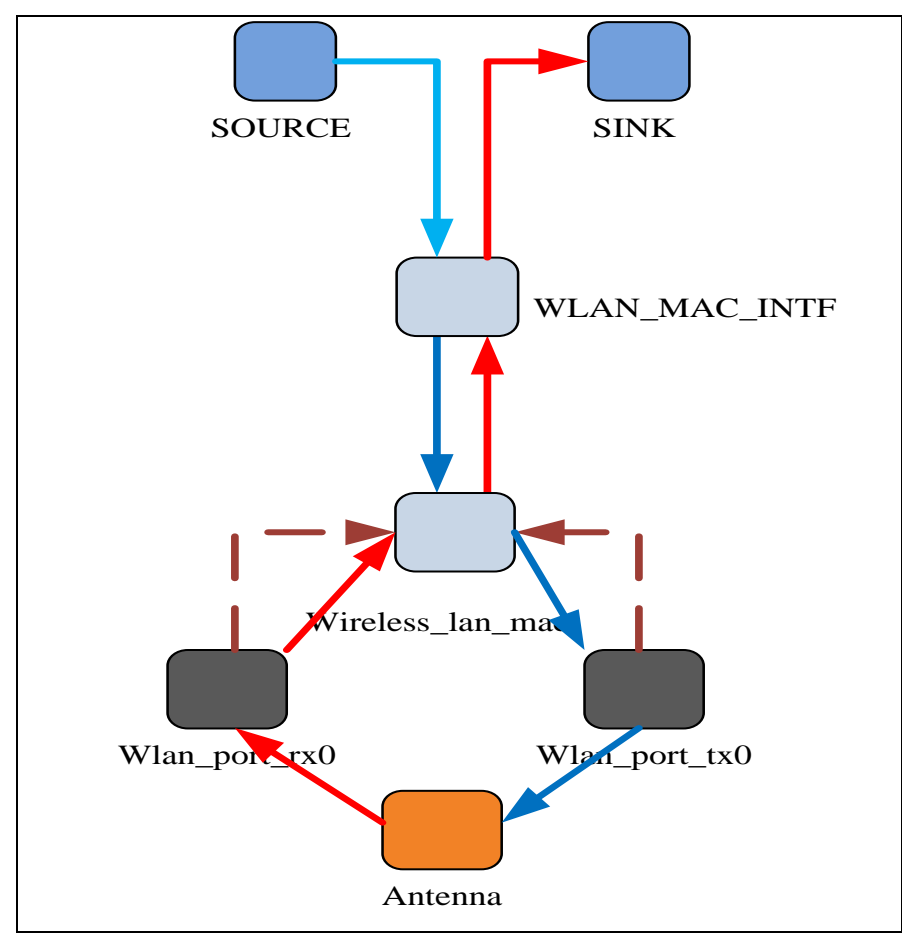

Figure. 2. Structure of node model

We design two scenarios, for linear and random topology. In linear topology, the all nodes in the network resides in single line as Figure. 3. In the random topology the nodes of the network randomly distributed in $100 \mathrm{~m} 2$ area. Figure. 4 depicts the linear topology scenario. Each simulation scenario contains 50 nodes, distributed in $500 \times 500 \mathrm{~m} 2$ area. The half node in the network is sender node and the half one is receiver. Each simulation runs for $600 \mathrm{~s}$. The result is the average of 10 runs with random seeds.

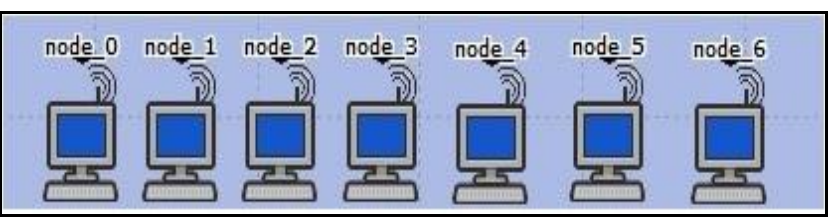

Figure. 3. Scenario of linear topology 


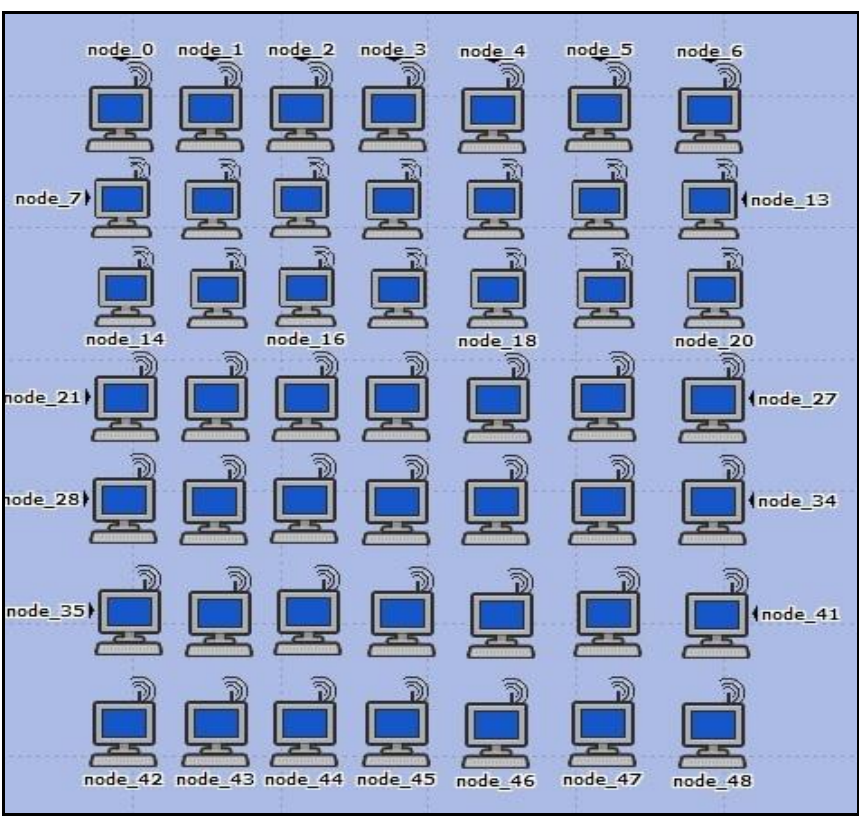

Figure. 4. Scenario of random topology

\section{PERFORMANCE RESULTS}

In this section we discuss the performance results of our simulation in terms of throughput and delay. Figure. 5 and Error! Reference source not found. depicts the simulation results of linear and random topology. Figure. 5 shows the simulation results of the throughput versus total offer load. In the figure, the performance of random topology is better than that of linear topology because in random topology the nodes of the network randomly distributed. Therefore the network gets better spatial reuse. But in the case of linear topology the nodes are in a straight line, so communication between two nodes block large area of the network due their higher transmission range, as a result overall performance of the network degrades.

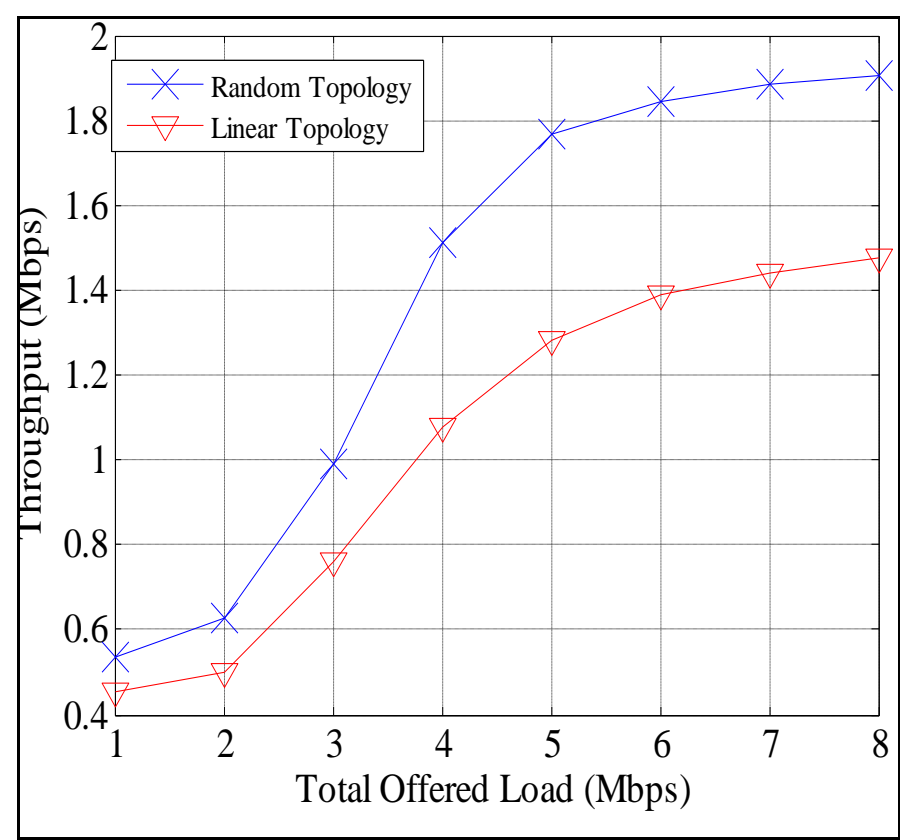

Figure. 5. Throughput versus Offered Load

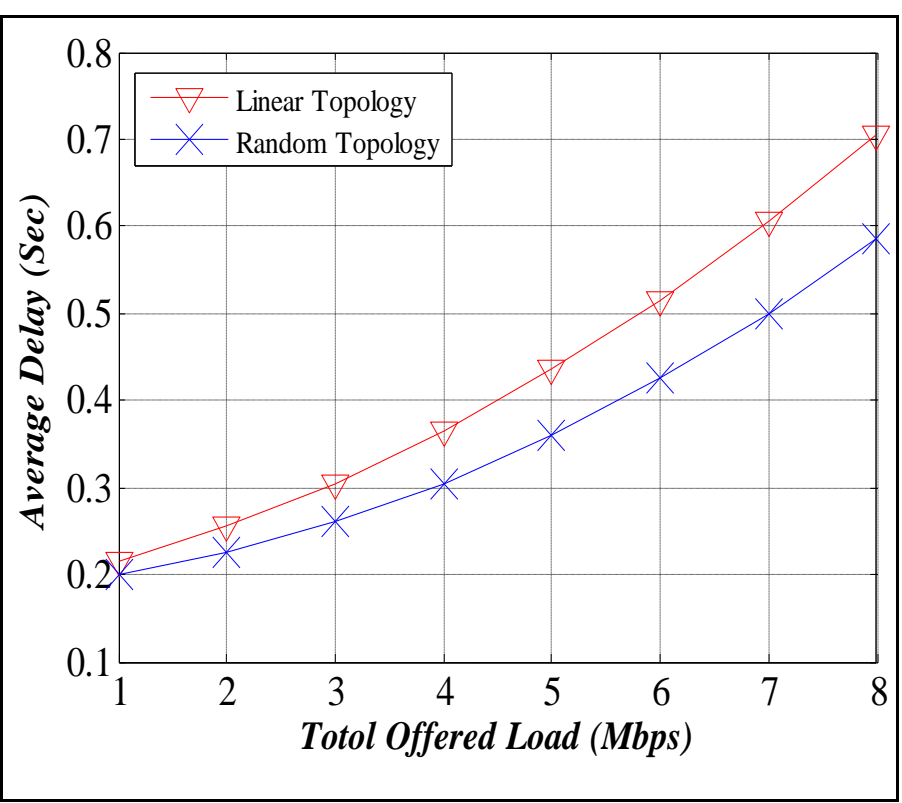

Figure. 6. Average Delay versus Offered Load

Error! Reference source not found. shows the simultion results of average delay versus total offered load in the network. In figure we can see that the linear topology result is poor than that of random topology. The reason is that, in linear topology the large portion of the network blocks, when a communication going on due to higher transmission range. Therefore, the neighbour node take long BO time, as a result the delay is increased.

\section{CONCLUSION}

This paper evaluates performance of the CDR-MAC protocol in terms of throughput and delay, by using OPNET simulation tool. We found the performance of random topology is better than that of linear topology. The simulation results indicate that if we use directional antenna, the overall throughput is increases with random topology in the network. But it not gives more benefit with linear topology because of its higher transmission range. The results validate that the use of directional antenna increases the spatial reuse.

\section{ACKNOWLEDGEMENT}

This work was supported by college of Medicine and College of Nursing (E-Learning Unit), King Saud University, Riyadh, Saudi Arabia and College of Computer Science, Jazan University, Jazan, Saudi Arabia.

\section{REFEREBCES}

[1] "Draft Supplement to Standard [for] Information Technology Telecommunications and information exchange between systems Local and metropolitan area networks Specific requirements Part 11: Wireless LAN Medium Access Control (MAC) and Physical Layer (PHY) spe,” IEEE Std P802.11g/D8.2. 2003.

[2] B. O'hara and A. Petrick, IEEE 802.11 handbook: a designer's companion. IEEE Standards Association, 2005 
[3] T. Korakis, G. Jakllari, and L. Tassiulas, "CDR-MAC: A protocol for full exploitation of directional antennas in ad hoc wireless networks," Mob. Comput. IEEE Trans., vol. 7, no. 2, pp. 145-155, 2008.

[4] R. R. Choudhury, X. Yang, R. Ramanathan, and N. H. Vaidya, "Using directional antennas for medium access control in ad hoc networks," in Proceedings of the 8th annual international conference on Mobile computing and networking, 2002, pp. 59-70.

[5] A. Nasipuri, S. Ye, J. You, and R. E. Hiromoto, "A MAC protocol for mobile ad hoc networks using directional antennas," Wireless Communications and Networking Confernce, 2000. WCNC. 2000 IEEE, vol. 3. pp. 1214 1219 vol.3, 2000.

[6] Y.-B. Ko, V. Shankarkumar, and N. H. Vaidya, "Medium access control protocols using directional antennas in ad hoc networks," INFOCOM 2000. Nineteenth Annual Joint Conference of the IEEE Computer and Communications Societies. Proceedings. IEEE, vol. 1. pp. 13-21 vol.1, 2000.

[7] M. Takata, M. Bandai, and T. Watanabe, "A MAC Protocol with Directional Antennas for Deafness Avoidance in Ad Hoc Networks," Global Telecommunications $\quad 2007$. GLOBECOM '07. IEEE. pp. 620-625, 2007.

[8] T. Korakis, G. Jakllari, and L. Tassiulas, "A MAC protocol for full exploitation of directional antennas in ad-hoc wireless networks," in Proceedings of the 4th ACM international symposium on Mobile ad hoc networking \&amp; computing, 2003, pp. 98-107.

[9] G. Jakllari, I. Broustis, T. Korakis, S. V Krishnamurthy, and L. Tassiulas, "Handling asymmetry in gain in directional antenna equipped ad hoc networks," Personal, Indoor and Mobile Radio Communications, 2005. PIMRC 2005. IEEE 16th International Symposium on, vol. 2. pp. 1284-1288 Vol. 2, 2005.

[10] H. Gossain, C. Cordeiro, T. Joshi, and D. P. Agrawal, "Cross-layer directional antenna MAC protocol for wireless ad hoc networks," Wirel. Commun. Mob. Comput, vol. 6, no. 2, pp. 171-182, 2006.

[11] O. Bazan and M. Jaseemuddin, "A Survey on MAC Protocols for Wireless Adhoc Networks with Beamforming Antennas," Communications Surveys \& Tutorials, IEEE, vol. 14, no. 2. pp. 216-239, 2012.

[12] G. Jakllari, I. Broustis, T. Korakis, S. V Krishnamurthy, and L. Tassiulas, "Handling asymmetry in gain in directional antenna equipped ad hoc networks," Personal, Indoor and Mobile Radio Communications, 2005. PIMRC 2005. IEEE 16th International Symposium on, vol. 2. pp. 1284-1288 Vol. 2, 2005.

[13] "OPNET 14.5."
http://www.opnet.com 